\title{
Presentation patterns and outcome of gliomatosis cerebri
}

\author{
GUSTAVO G. RAJZ ${ }^{1}$, DVORA NASS ${ }^{2}$, ELISA TALIANSKI $^{3}$, RAPHAEL PFEFFER ${ }^{3}$, \\ ROBERTO SPIEGELMANN ${ }^{1}$ and ZVI R. COHEN ${ }^{1}$
}

\author{
Departments of ${ }^{1}$ Neurosurgery, ${ }^{2}$ Neuropathology, and ${ }^{3}$ Neuro-Oncology, The Chaim Sheba Medical Center, \\ Tel-Hashomer, Sackler School of Medicine, Tel Aviv University, Tel Aviv, Israel
}

Received July 5, 2011; Accepted September 23, 2011

DOI: $10.3892 / \mathrm{ol} .2011 .445$

\begin{abstract}
A total of 25 patients with gliomatosis cerebri (19 males and 6 females; median age 51 years, range 10-73 years) were diagnosed and treated at the Sheba Medical Center between 1995 and 2009. Of these, 3 patients were 10 years old at the time of diagnosis. Seizures were the initial clinical presentation in 19 patients, focal signs in 16 patients, headaches in 7 patients, cognitive disorder in 4 patients and rapidly progressive hemiparesis in 1 patient. Magnetic resonance imaging (MRI) was performed in the patients and demonstrated a diffuse infiltrative process with a hyperintensity signal on T2-weighted images and a minimal mass effect. Some level of enhancement on MRI was observed in 6 patients. The infiltrative process involved at least two lobes in each patient. Biopsy was performed for diagnosis in the majority of patients. In 1 patient with a markedly rapid deterioration, the diagnosis was established at autopsy. The pathology was compatible with gliomatosis with a diffuse infiltrative low-grade astrocytoma in 21 patients and anaplastic astrocytoma in 5 patients. The patients were treated with whole-brain radiation therapy and 7 patients were treated with combined whole-brain radiation therapy and chemotherapy. Treatment appeared to stabilize 6 patients or improve the clinical condition in 7 patients. Due to the small number of patients in the present study, however, further studies are required to determine the effect of treatment on the natural history of the disease.
\end{abstract}

\section{Introduction}

Gliomatosis cerebri (GC) is a rare primary diffuse brain tumor first described by Nevin (1) in 1938. According to the World Health Organization classification, GC is recognized as a specific entity among neuroepithelial tumors of uncertain origin (2). The definition of GC as a separate entity is questionable, however, and certain authors consider GC to be one end of the spectrum of diffusely infiltrating astrocytomas (3). Kattar et al (4) performed a clonal analysis of gliomas and concluded that GC begins as an oligoclonal process or may

Correspondence to: Dr Zvi R. Cohen, Department of Neurosurgery, Sheba Medical Center, Tel-Ashomer 52621, Israel

E-mail: zvi.cohen@sheba.health.gov.il

Key words: gliomatosis, brain tumor result from collision gliomas, in contrast to the monoclonality usually observed in low grade and malignant gliomas. GC usually involves at least two lobes of the brain and histologic evaluation reveals a diffuse infiltrative low-grade astrocytoma. The presenting symptoms of GC in the literature are nonspecific. Magnetic resonance imaging (MRI) studies indicate a diffuse infiltrative process, particularly on T2 images, and the diagnosis is made by a stereotactic or open biopsy. There is currently no effective treatment for GC. Surgical treatment of GC is limited due to the extensive diffuse infiltrative process; therefore, treatment generally comprises anticonvulsants and steroids. The literature contains several anecdotal reports of radiation therapy (5-11). Despite treatment, the prognosis is poor. Ross et al (11) reviewed several series with median survival ranging between 6 and 39 months, but certain patients with GC have survived for a longer period of time (6).

\section{Patients and methods}

Patients. Between 1995 and 2009, 25 patients with GC (19 males, 6 females; mean age 51 years, range 10-73 years) were diagnosed and treated at the Sheba Medical Center, Israel. A total of 3 patients were 10 years old at the time of diagnosis. A retrospective analysis was performed. Patient characteristics and follow-up data were obtained from clinical charts. Histologic specimens and MRI studies were reviewed and the diagnosis was based on characteristic histologic and radiologic findings.

Methods. MRI of the brain was performed for all patients. The radiologic criteria were diffuse infiltrative tumor with involvement of at least two lobes (with or without corpus callosum infiltration). Diagnosis was confirmed by stereotactic or open biopsy except in the case of 1 patient with rapid deterioration, who was diagnosed in a postmortem study. The patients (with the exception of the one who exhibited rapid deterioration) were treated with whole brain radiation therapy (WBRT). A total of 7 patients were treated with combined WBRT and chemotherapy. Outcome was determined by post-irradiation MRI, neurologic status, performance status and survival.

\section{Results}

Presenting symptoms of patients. Seizures were the initial clinical presentation in 19 patients, focal signs in 16 patients, 
headaches in 5 patients, cognitive changes in 7 patients, and rapidly progressive hemiparesis in 1 patient. MRI revealed a diffuse infiltrative process observed as a high-intensity signal on T2-weighted images with minimal mass effect. The infiltrative process involved at least two lobes.

A total of 9 patients exhibited involvement of the corpus callosum. In the majority of the patients (19 of 25), the gliomatosis infiltrated the right hemisphere. A total of 9 patients had bihemispheric involvement. Some level of enhancement on MRI was observed in 6 patients.

Biopsy outcomes. The diagnosis was confirmed histologically by a stereotactic biopsy in the majority of patients and by open biopsy in a small number of patients. Markedly rapid deterioration was observed in one patient for whom diagnosis was established at autopsy. The patients were treated with WBRT, 1 patient was treated with pre-irradiation chemotherapy, and 6 patients were treated with combined therapy. Survival time from diagnosis for the patients $(\mathrm{n}=25)$ was between 11 days and 42 months (median survival 16.7 months).

Patient survival. The 17 patients who were treated with WBRT alone had a median survival of 13.7 months (range 1-35). The 7 patients who were treated with combined whole brain radiation therapy and chemotherapy had a median survival of 26.14 months (range 6-42). Treatment appeared to stabilize 6 patients. The 3 10-year-old patients were treated with WBRT, which resulted in temporary clinical stabilization, succumbed to the disease approximately 1 year after the diagnosis was established. A total of 9 adults rapidly deteriorated after completing treatment and survived less than one year from the time of diagnosis. One patient was not treated at all. Treatment appeared to improve the clinical condition in 9 patients with a survival range of 20-42 months (Table I).

\section{Discussion}

We present the patient characteristics, presenting symptoms, radiologic patterns, and outcome following treatment in 25 patients with GC. In a retrospective review of the literature (160 cases in 85 reports), Jennings et al (12) reported that the peak incidence of GC is between 40 and 50 years of age and that GC is extremely rare in the senior population, consistent with our series (mean age of 51 years). Jennings et al (12) reported an age range between the neonatal period and 83 years of age. The oldest patient in our cohort was 73 years of age. In contrast to published data indicating that older age is a poor prognostic factor for glioblastoma and that low-grade astrocytoma and juvenile pilocytic astrocytoma in the juvenile population have a favorable prognosis, the 3 children in our series and the neonate reported by Jennings et al (12) had a median survival of only approximately 1 year after diagnosis and the disease course of the 73-year-old patient was stabilized following WBRT. The difference in outcome may support the hypothesis that $\mathrm{GC}$ is a separate entity from diffuse infiltrative astrocytoma. Our series demonstrated a male:female ratio of 19:6, and in contrast to other studies, showed that both males and females are equally affected $(5,12)$. In our series, the majority of patients presented with seizures or focal neurologic signs. Only a small proportion of our patients presented with headaches. Jennings et al (12) reported 3 pediatric cases with intractable epilepsy as the presenting symptom of GC. In that study, 160 cases were retrospectively reviewed and an attempt to determine the natural history of GC was performed. The common neurologic symptoms and signs included corticospinal tract deficits (58\%), cognitive changes $(44 \%)$, headache (39\%), seizures (38\%), cranioneuropathies $(37 \%)$, increased intracranial pressure (ICP) (34\%), and spinocerebellar deficits (33\%). Our series is consistent with other reports documenting seizures as the main presenting symptom (13-15). However, other studies have indicated that headaches and elevated ICP are more common presenting symptoms $(11,16-18)$. CruzVelarde et al (19) reported a patient with GC who presented with headaches and bilateral sixth cranial nerve palsy with elevated ICP confirmed by invasive ventricular monitoring.

Due to the infiltrative nature of the tumor and the lack of a significant mass effect, it is reasonable to expect seizures to be the main presenting symptom rather than signs of elevated ICP, as shown in our series. Two types of GC have been distinguished (20). Type 1 is a classical discrete lesion in which there is diffuse neoplastic growth and enlargement of the involved existing structures without a solid tumor component. Type $2 \mathrm{GC}$, by contrast, is characterized by a clear neoplastic mass in addition to a diffuse lesion. The majority of the patients in our series had a Type $1 \mathrm{GC}$ diffuse lesion without evidence of enhancement or a circumscribed tumor mass. Behavioral, personality and cognitive changes, reported by other authors $(10,20,21)$, were not common in our patients $(4 / 22)$ or in other reported series (15). In our patients, the cognitive and personality changes may be associated with the bifrontal location of the GC.

In our series, the tumor involved at least two lobes of the brain with a hyperintensity signal in T2-weighted images in all patients and involvement of the corpus callosum in certain patients. This is consistent with previous publications $(9,11,15,22,23)$. High signal intensity in the white matter on T2-weighted images reflects spreading of the infiltrative tumor or secondary destruction of the myelin fibers (17). The relative preservation of the blood-brain behavior is the most likely reason for nonenhancement on post-contrast T1-weighted images $(7,24,25)$. Shin et al $(26)$ compared MRI to computed tomography scans in 9 patients with GC and revealed that the extent of the disease was evaluated significantly more favorably on T2-weighted MR images than on T1-weighted MR images and computed tomography scans. It is now common practice to use MRI in the initial evaluation of GC. Our data and data published by other authors $(5,9,15)$ support this approach. Bendszus et al (27) evaluated 8 patients with GC who underwent MRI and MR spectroscopy and revealed that biopsy specimens from regions with maximally elevated ratio levels of choline/ $\mathrm{N}$-acetylaspartate showed dense infiltration of tumor cells. Their conclusion was that MR spectroscopy may be used to classify GC as a stable or progressive disease and thus has potential therapeutic value. Shintani et al (28) presented a case report of positron emission tomography (PET) changes in a patient with GC treated with radiation. ${ }^{11} \mathrm{C}$-Methionine PET images showed hypermetabolism, whereas ${ }^{15} \mathrm{O}$-water PET images showed a marked increase in cerebral blood flow in GC lesions. However, the PET images revealed marked improvement 6 months subsequent to radiotherapy. 


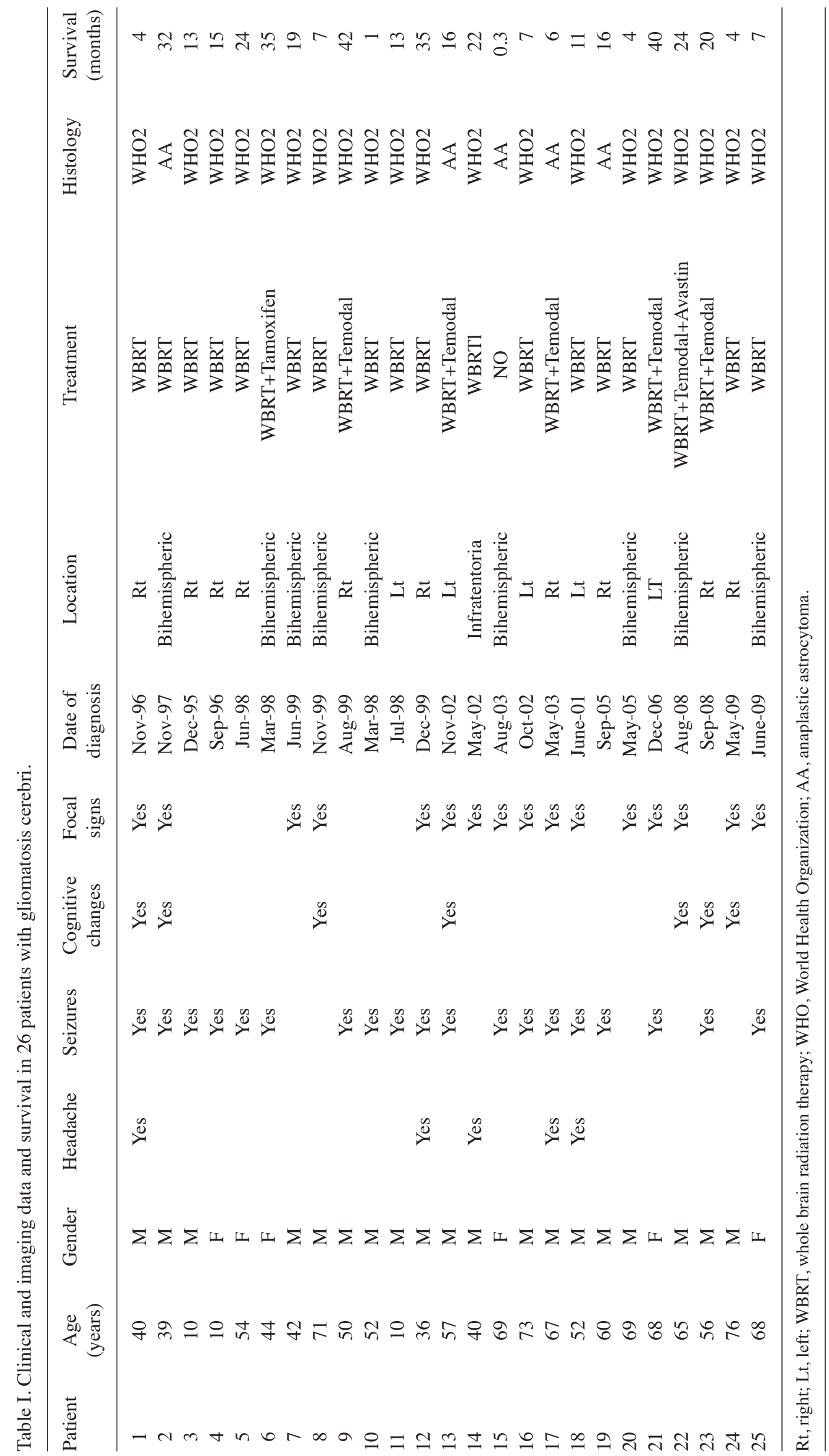


Kaloshi et al (29) showed that a gray matter index has prognostic value in patients with GC treated with upfront chemotherapy (PCV, temozolomide). Ellingson et al (30) created functional diffusion maps (fDMs) to examine changes in the apparent diffusion coefficient calculated from serial diffusion-weighted images of abnormal FLAIR signal intensity in a patients diagnosed with GC. These authors demonstrated that the absolute volume of hypercellularity extracted from fDMs was useful for tracking tumor growth, which correlated in time with a progressive decline in neurologic status despite the lack of changes in traditional MR images.

Due to the diffuse infiltrative pattern of the disease, the limitation of surgical resection is clear and radiation and chemotherapy are the only potential therapies. The literature contains a number of anecdotal reports of radiation therapy as treatment. Artigas et al (6) presented two cases with transient improvement following radiation. The patients subsequently deteriorated and succumbed to their disease at 8 and 12 months, respectively. Rippe et al presented a 16-year-old patient with 1 year of survival following treatment with local-field treatment of $54 \mathrm{~Gy}$ (7). Schober et al (8) presented 2 patients with GC treated with radiation: 1 succumbed to pulmonary embolus during treatment and the other deteriorated during WBRT and succumbed 5 months after diagnosis. Cozad et al (31) presented 3 patients with GC treated with WBRT at a dose of 54 Gy; 1 patient deteriorated during treatment, 1 demonstrated brief stabilization following treatment prior to deterioration, and 1 exhibited reversal of the clinical signs and symptoms with stability and favorable quality of life at 16 months following diagnosis. One large retrospective study emphasized the significance of radiation therapy in patients with GC. Kim et al (9) presented a series of 14 patients diagnosed with GC. The median survival following WBRT (mean 5780 cGy) was 38.4 months after diagnosis. Horst et al (5) reviewed data from previous publications beginning in 1985 on 17 patients with GC who underwent radiation therapy. These patients received a median dose of 51.2 Gy (22-64.8 Gy). Median survival was 22.8 months (8-42 months). The authors concluded that radiation therapy in GC is associated with a temporary improvement in or stabilization of clinical symptoms in the majority of patients. Elshaikh et al (32) presented 8 of 12 patients who received radiation treatment (median dose of $55.4 \mathrm{~Gy}$ ). The clinical and radiological findings improved in 3 patients, stabilized in 3 patients, and deteriorated in 2 patients. Median survival was 11.4 months and the authors concluded that radiation alone was not sufficient and therefore more aggressive therapy may be needed.

A review of the literature of recent years reveals efforts to combine chemotherapy and radiation therapy $(33,34)$ or to use chemotherapy alone (35). Vates et al (34) treated only 3 patients with radiation and there was no significant difference in outcome compared to 13 patients who underwent radiation therapy alone.

Seiz et al (36) reported a small number of patients with overexpression of factors such as vascular endothelial growth factor or cyclooxygenase-2. The patients were treated with temozolomide and celecoxib as antiangiogenic treatment following external radiotherapy and there was no disease progression for at least 6 months based on a follow-up MRI.
The authors concluded that low-dose chemotherapy may provide a promising approach for treating these patients.

Sanson et al (35) analyzed 296 individual cases (90 patients followed through the ANOCEF network, and 206 cases from the literature) and concluded that despite a high rate of stabilization, the survival impact of WBRT, which carries the risk of severe toxicity, remains unclear. The up-front chemotherapy benefit to certain patients may be preferred to WBRT. Due to the many biases of such retrospective heterogeneous data, multicenter clinical trials of this rare disease should be conducted.

Ware et al (33) performed comparative genomic hybridization in 22 patients with GC. Loss of chromosomes 13q and $10 \mathrm{q}$ and gains of $7 \mathrm{q}$ were independent significant predictors of poor survival ( $\mathrm{P}=0.0032,0.0335$, and 0.0487 , respectively). Contrast enhancement was the most significant predictor of poor survival $(\mathrm{P}=0.0026)$. These authors concluded that the presence of these aberrations and of any contrast enhancement on MRI scans are possible stratifiers for patients with GC. Stratification of GC into higher- and lower-grade forms may be beneficial for tailoring treatments to patients with this disease.

D'Urso et al (37) investigated gene expression profiling in 59 patients with GC and identified a 23-gene signature that was capable of predicting patient prognosis. D'Urso et al (38) presented a case report with overexpression of vascular cell adhesion molecule 1 and vascular endothelial growth factor, indicating that a high degree of neovascularization was associated with a poor prognosis.

In the present study, we presented 25 patients with GC, which is a relatively large series and compared them to previously published data. In our series following WBRT (50 Gy) the median survival was 14.8 months. The median survival of patients treated with combined treatment was 22.6 months (5 patients). Due to the small number of patients, the present statistical analysis is of limited value, but based on our experience and a review of the literature, WBRT may stabilize the clinical condition and reverse neurologic signs in some patients with GC.

\section{References}

1. Nevin S: Gliomatosis Cerebri. Brain 61: 170-191, 1938.

2. Louis DN, Ohgaki H, Wiestler OD and Cavenee WK: WHO Classification of Tumors, Pathology and Genetics of Tumors Of the Nervous System. International Agency for Research on Cancer, 69372, Lyon, France, Lyon, 2000.

3. Fallentin E, Skriver E, Herning M and Broholm H: Gliomatosis cerebri - an appropriate diagnosis? Case reports. Acta Radiol 38: 381-390, 1997.

4. Kattar MM, Kupsky WJ, Shimoyama RK, et al: Clonal analysis of gliomas. Hum Pathol 28: 1166-1179, 1997.

5. Horst E, Micke O, Romppainen ML, et al: Radiation therapy approach in gliomatosis cerebri - case reports and literature review. Acta Oncol 39: 747-751, 2000.

6. Artigas J, Cervos-Navarro J, Iglesias JR and Ebhardt G: Gliomatosis cerebri: clinical and histological findings. Clin Neuropathol 4: 135-148, 1985.

7. Rippe DJ, Boyko OB, Fuller GN, Friedman HS, Oakes WJ and Schold SC: Gadopentetate-dimeglumine-enhanced MR imaging of gliomatosis cerebri: appearance mimicking leptomeningeal tumor dissemination. AJNR Am J Neuroradiol 11: 800-801, 1990.

8. Schober R, Mai JK, Volk B and Wechsler W: Gliomatosis cerebri: bioptical approach and neuropathological verification. Acta Neurochir 113: 131-137, 1991. 
9. Kim DG, Yang HJ, Park IA, et al: Gliomatosis cerebri: clinical features, treatment, and prognosis. Acta Neurochir 140: 755-762, 1998.

10. Felsberg GJ, Silver SA, Brown MT and Tien RD Radiologic-pathologic correlation. Gliomatosis cerebri. AJNR Am J Neuroradiol 15: 1745-1753, 1994.

11. Ross IB, Robitaille Y, Villemure JG and Tampieri D: Diagnosis and management of gliomatosis cerebri: recent trends. Surg Neurol 36: 431-440, 1991

12. Jennings MT, Frenchman M, Shehab T, et al: Gliomatosis cerebri presenting as intractable epilepsy during early childhood. J Child Neurol 10: 37-45, 1995.

13. Gottesman M, Laufer H and Patel M: Gliomatosis cerebri: a case report. Clin Neuropathol 10: 303-305, 1991.

14. Bertrand E, Kryst-Widzgowska T and Baranska M: Gliomatosis cerebri at the 17-year-old girl - correlations of histological, CT and MRI appearance. Folia Neuropathol 32: 245-249, 1994.

15. Del Carpio-O'Donovan R, Korah I, Salazar A and Melancon D: Gliomatosis cerebri. Radiology 198: 831-835, 1996.

16. Balakrishnan V, Hornabrook RW and Alexander WS: Gliomatosis cerebri - report of a case. Pathology 17: 123-126, 1985.

17. Spagnoli MV, Grossman RI, Packer RJ, et al: Magnetic resonance imaging determination of gliomatosis cerebri. Neuroradiology 29: 15-18, 1987.

18. Kandler RH, Smith CM, Broome JC and Davies-Jones GA Gliomatosis cerebri: a clinical, radiological and pathological report of four cases. Br J Neurosurg 5: 187-193, 1991.

19. Cruz-Velarde JA, Munoz L, Rodrigalvarez R and Grandas F: [Intracranial hypertension as the first clinical manifestation of gliomatosis cerebri]. Neurologia 15: 32-34, 2000.

20. Pyhtinen J and Paakko E: A difficult diagnosis of gliomatosis cerebri. Neuroradiology 38: 444-448, 1996.

21. Dickson DW, Horoupian DS, Thal LJ and Lantos G: Gliomatosis cerebri presenting with hydrocephalus and dementia. AJNR Am J Neuroradiol 9: 200-202, 1988.

22. Choi D, Schulz U and Seex K: Gliomatosis cerebri: a brain tumour which is too difficult to treat? Scott Med J 43: 84-86, 1998.

23. Borgis KJ, Larsen JL, Mork S and Bruckmann H: [Clinical and neuroradiological findings in gliomatosis of the brain with special reference to magnetic resonance tomography]. Klin Padiatr 203: 345-349, 1991 .

24. Hayek $\mathrm{J}$ and Valavanis A: Computed tomography of gliomatosis cerebri. Comput Radiol 6: 93-98, 1982.
25. Yanaka K, Kamezaki T, Kobayashi E, Matsueda K, Yoshii Y and Nose T: MR imaging of diffuse glioma. AJNR Am J Neuroradiol 13: 349-351, 1992.

26. Shin YM, Chang KH, Han MH, et al: Gliomatosis cerebri: comparison of MR and CT features. AJR Am J Roentgenol 161: 859-862, 1993.

27. Bendszus M, Warmuth-Metz M, Klein R, et al: MR spectroscopy in gliomatosis cerebri. AJNR Am J Neuroradiol 21: 375-380, 2000.

28. Shintani S, Tsuruoka S and Shiigai T: Serial positron emission tomography (PET) in gliomatosis cerebri treated with radiotherapy: a case report. J Neurol Sci 173: 25-31, 2000.

29. Kaloshi G, Guillevin R, Martin-Duverneuil N, et al: Gray matter involvement predicts chemosensitivity and prognosis in gliomatosis cerebri. Neurology 73: 445-449, 2009.

30. Ellingson BM, Rand SD, Malkin MG and Schmainda KM: Utility of functional diffusion maps to monitor a patient diagnosed with gliomatosis cerebri. J Neurooncol Oct 8, 2009 (e-pub ahead of print)

31. Cozad SC, Townsend P, Morantz RA, Jenny AB, Kepes JJ and Smalley SR: Gliomatosis cerebri. Results with radiation therapy. Cancer 78: 1789-1793, 1996.

32. Elshaikh MA, Stevens GH, Peereboom DM, et al: Gliomatosis cerebri: treatment results with radiotherapy alone. Cancer 95: 2027-2031, 2002

33. Ware ML, Hirose Y, Scheithauer BW, et al: Genetic aberrations in gliomatosis cerebri. Neurosurgery 60: 150-158, 2007.

34. Vates GE, Chang S, Lamborn KR, Prados M and Berger MS: Gliomatosis cerebri: a review of 22 cases. Neurosurgery 53: 261-271, 2003.

35. Sanson M, Cartalat-Carel S, Taillibert S, et al: Initial chemotherapy in gliomatosis cerebri. Neurology 63: 270-275, 2004.

36. Seiz M, Kohlhof P, Brockmann MA, et al: First experiences with low-dose anti-angiogenic treatment in gliomatosis cerebri with signs of angiogenic activity. Anticancer Res 29: 3261-3267, 2009.

37. D'Urso OF, D'Urso PI, Marsigliante S, et al: Correlative analysis of gene expression profile and prognosis in patients with gliomatosis cerebri. Cancer 115: 3749-3757, 2009.

38. D'Urso PI, D'Urso OF, Marsigliante S, et al: Gliomatosis cerebri type II: two case reports. J Med Case Rep 3: 7225, 2009. 